\title{
Vulcanoid Asteroids and Sun-Grazing Comets - Past Encounters and Possible Outcomes
}

\author{
Martin Beech ${ }^{1,2}$, Lowell Peltier ${ }^{2}$ \\ ${ }^{1}$ Campion College, The University of Regina, Regina, SK, Canada \\ ${ }^{2}$ Department of Physics, The University of Regina, Regina, SK. Canada
}

\section{Email address:}

beechm@uregina.ca (M. Beech), peltie21@uregina.ca (L. Peltier)

\section{To cite this article:}

Martin Beech, Lowell Peltier. Vulcanoid Asteroids and Sun-Grazing Comets - Past Encounters and Possible Outcomes. American Journal of Astronomy and Astrophysics. Vol. 3, No. 2, 2015, pp. 26-36. doi: 10.11648/j.ajaa.20150302.12

\begin{abstract}
The region between 0.07 to 0.25 au from the Sun is regularly crossed by sungrazing and small perihelion distance periodic comets. This zone also supports stable orbits that may be occupied by Vulcanoid asteroids. In this article we review the circumstances associated with those comets known to have passed through the putative Vulcanoid region, and we review the various histories associated with a sub-group of these comets that have been observed to displayed anomalous behaviors shortly before or after perihelion passage. In all 406 known comets are found to have passed through the Vulcanoid zone; the earliest recorded comet to do so being C/400 F1, with comet C/2008 J13 (SOHO) being the last in the data set used (complete to 2014). Only two of these comets, however, are known to be short period comets, C/1917 F1 Mellish and 96P / Machholz 1, with the majority being sungrazing comets moving along parabolic orbits. We examine the case history of comet C/1917 F1 Mellish in some detail since numerical simulations suggest that over the past $\sim 40$ thousand years it has regularly passed through the Vulcanoid zone. Additionally, this particular comet is linked to the December Monocerotid meteor shower, which is known to have produced a series of very bright fireball displays in the $11^{\text {th }}$ Century. An extremely small impact probability of order $10^{-19}$ per perihelion passage with a Vulcanoid of diameter $1 \mathrm{~km}$ or larger is determined for comet Mellish, and we conclude that the ancient fireball display is not likely associated with a Vulcanoid collision. Indeed, while we find no evidence to indicate that any historical collisions between a cometary nucleus and a Vulcanoid have occurred, this result, we suggest, does not automatically mean that no Vulcanoids exist at the present time, or that collisions have not taken place in the past. Likewise, these results do not rule out the possibility of collisions being observable at future times. As ever, since first being hypothesized, if they exist at all, the Vulcanoid asteroids remain elusive.
\end{abstract}

Keywords: Vulcanoids, Cometary Impacts, Cometary Outbursts, Meteor Showers

\section{Introduction}

Cometary nuclei have long been recognized as crystalline time capsules that contain important information about the initial chemistry and dynamical history of the early solar system [1]. In a complementary and contra sense, however, cometary nuclei can also be thought of as probes to those regions of the solar system that are inaccessible to direct observation from Earth and/or in situ spacecraft study. The idea being exploited here is that cometary nuclei can betray the existence of other bodies within the solar system through the action of collisions. Such collisions it is asserted being able to potentially trigger observable and unexpected outburst activity from the comet and possibly from within any associated Earth-intercepting meteoroid streams [2].
Starting from the basis of a known cometary orbit, therefore, the identification of collision generated outburst phenomenon can, at least in principle, be used to betray the presence of interplanetary objects passing through the perihelion to aphelion region of a specific comet's orbital plane.

The discovery of the main-belt population of comets $[3,4]$ affords one example where not only was an unexpected reservoir of cometary nuclei discovered, but the discovery outbursts themselves were governed by impact events although we note that thermal forcing, and rotational spin-up are additional trigger mechanisms. The remarkable discovery images of P/2010 A2 LINEAR further exemplifies the effects of what appears to be a collision between a main-belt cometary nucleus and a multiple-tens-of-meter diameter main-belt asteroid [5]. Comets not belonging to the main-belt population have also been known to undergo outbursts while 
passing through the main-belt asteroid region [6], and this activity, in many cases, was likely driven by impacts associated with meter-sized asteroid fragments.

In general the likelihood of a collision occurring between a cometary nucleus and a smaller-sized impactor is maximized in those regions were the impactors reside on long-lived, stable orbits - such as in the main-belt asteroid region. For Jupiter family comets, therefore, collisions are most likely going to take place when they are close to aphelion. Alternatively, however, collisions close to perihelion might occur for both long period and Jupiter family comets, sungrazers, and Halley type comets when they pass through the inter-Mercurial region where the long-suspected population of Vulcanoid asteroids is reasoned to reside $[7,8$, $9,10,11]$. Although no member of the Vulcanoid asteroid family has (so far) been detected (see section 2 below), the rational in this review is to investigate the history of outburst behavior associated with those comets known to have passed through the putative Vulcanoid zone. While it is recognized that comets can fragment and undergo outburst activity for many different reasons $[12,13,14$,] one possible mechanism for such behaviors is that due to an impact with another body, and in this review the possibility that some of the potential impacting bodies could be Vulcanoid asteroids is investigated. As discussed in more detail in section 3, many known comets have a perihelion and/or a nodal point(s) located within the Vulcanoid region, and it is this putative population of objects that we discuss next.

\section{The Vulcanoids}

The Vulcanoids are a hypothesized class of asteroids that reside in a small annular region interior to the orbit of Mercury. Historically the inter-Mercurial planet Vulcan, and later, from about the mid- $20^{\text {th }}$ century onward, the Vulcanoid asteroids have been looked for at the times of total solar eclipse when the regions close to the Sun (specifically between $\sim 15$ and $\sim 55 \mathrm{R}_{\odot}$ ) can be examined for faint objects. Courten, Brown and Albert [15], for example, conducted a series of photographic searches during 6 solar eclipses in the 10 year time interval 1966 to 1976 , and while they reported the detection of several anomalous faint objects, located within 20 solar radii of the Sun, it is not clear whether any of these objects were Vulcanoids. Similar such sightings were made during the total solar eclipse of 29 July 1878 when James Watson (University of Michigan) along with renowned comet hunter Lewis Swift claimed to have observed an anomalous object which they argued was the planet Vulcan [16] - Vulcan in this case being the inter-mercurial planet hypothesized by Urbain Le Verrier, in 1859, to explain the advanced precession rate of Mercury's orbit [8]. More recently, the wide angle camera aboard the MESSENGER spacecraft was used to search for Vulcanoids prior to its orbit insertion about Mercury in March of 2011, but no anomalous objects brighter than a limiting visual magnitude of +8 (corresponding to sizes larger than $\sim 15 \mathrm{~km}$ across) were detected [17]. In principle it might be possible to infer the existence of at least a primordial population of Vulcanoids through the detailed analysis of the cumulative number of craters versus crater size distribution on Mercury [18] - the putative Vulcanoid impactors adding to the population of objects responsible for the cratering recorded elsewhere in the solar system. The complex volcanic history and surface evolution of Mercury, however, conspire to make such an analysis difficult, at best, and at present there is no clear-cut data to suggest that an excess of craters (or an extended cratering history) due to Vulcanoid impacts exists. A number of searches for Vulcanoids, with the SOHO and STEREO Sun-monitoring space-platforms, have been made during the past 15 years $[9,10,11]$ but, again, no Vulcanoid objects have been positively identified. These collective nullobservations, of course, cut two ways; either there are no such objects to be found, or, the size distribution and surface albedos of the Vulcanoids are such that their reflected-light values continue to fall below the threshold for detection with the available instrumentation. On purely dynamical grounds, it would be somewhat surprising if no Vulcanoid asteroids exist since most other regions within the solar system that allow for stable orbits appear to have known and identified populations of objects. A principle of mediocrity approach therefore suggests that it is the detection threshold condition that likely applies, and that a Vulcanoid population genuinely does exist but that it contains no large objects at the present epoch. Indeed, a search for Vulcanoids using the data archive generated by the STEREO Heliospheric Imager by Steffl et al. [11] reveals that there are no objects larger than about 6 $\mathrm{km}$ across within the present Vulcanoid population (for an assumed R-band albedo of 0.05 - a value characteristics of that derived for Mercury and the C-type asteroids). Steffl et al. [11] additionally estimate, assuming a steady-state collisional fragmentation history, that there are no more than 76 Vulcanoids larger than $1 \mathrm{~km}$ across at the present epoch.

While past searches have failed to reveal any Vulcanoid candidates there is a reasonable consensus on where, should they truly exist, such objects must reside. Numerical simulations conducted by Evans and Tabachnik [19, 20] indicate that a Vulcanoid stability zone exists between 0.09 and $0.20 \mathrm{au}$ from the Sun, with orbital inclinations typically in the range of about $5^{\circ}$, although inclinations as high as $10^{\circ}$ appear to be possible. These authors also find two reasonable strong mean-motion resonance clearing-zones at 0.15 au (due to Mercury) and 0.18 au (due to Venus). Based upon both dynamical and thermodynamic lifetime against sublimation arguments Campins et al. [21] suggest that the search area for Vulcanoids might best be confined to the ecliptic at distances between 0.1 and 0.25 au from the Sun. Steffl et al. [11] and Durda et al. [9] place the Vulcanoid inner and outer boundaries at 0.07 and 0.21 au from the Sun and allow for inclinations as high as $15^{\circ}$ away from the ecliptic. Stern and Durda [22] set a thermodynamic limit of 0.06 au for the inner boundary of the Vulcanoid region and allow orbits with inclinations up to $16^{\circ}$, while Vokrouhlicky et al. [23] adopt the radial limits set by Evans and Tabachnik [19]. Given the values adopted by previous researchers we assume in this 
analysis that a maximum Vulcanoid region can be defined (figure 1) as that zone extending from 0.06 to 0.25 au away from the Sun, with orbital inclinations reaching as high as $15^{\circ}$ to the ecliptic.

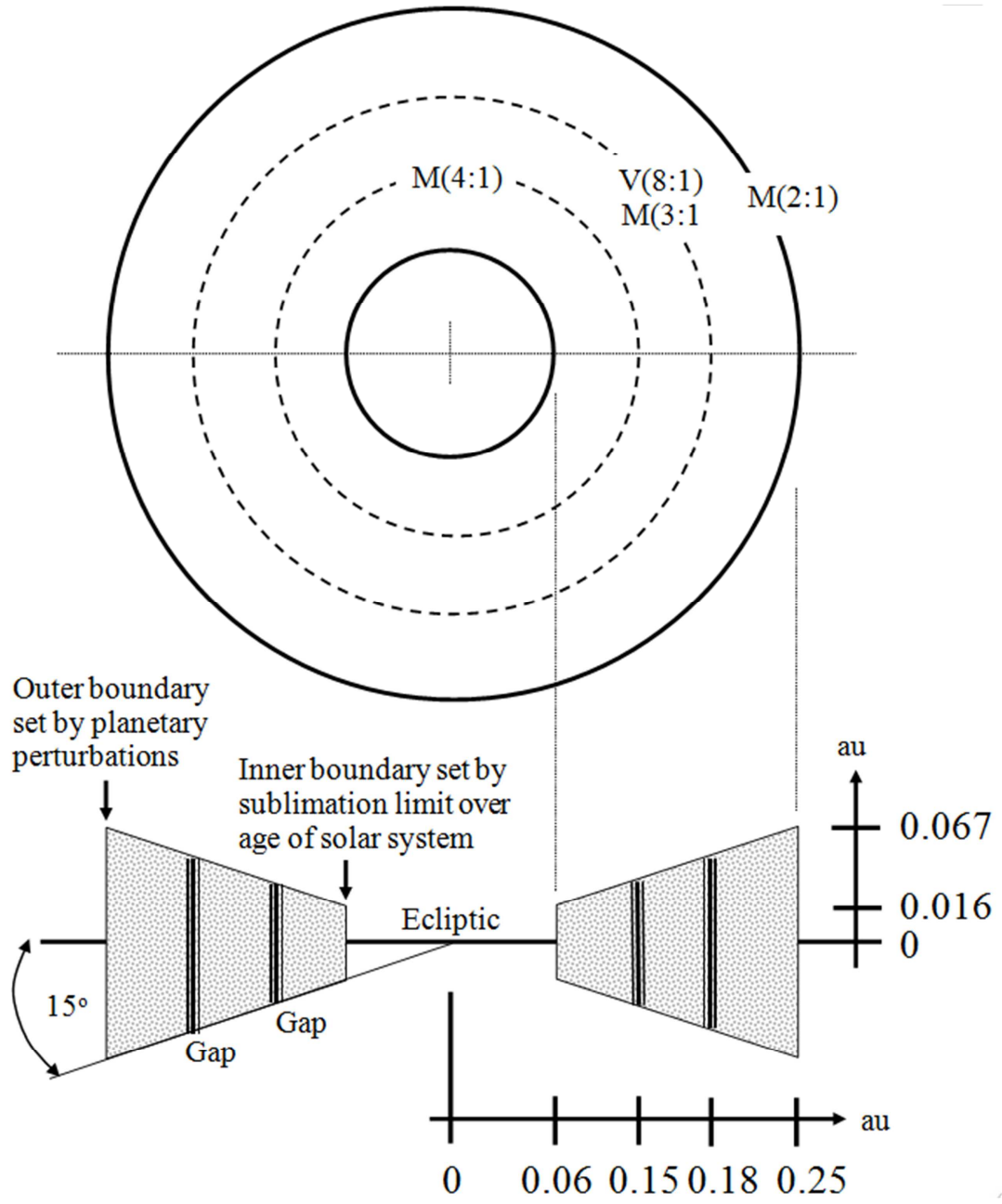

Figure 1. The maximum Vulcanoid zone. This schematic diagram illustrates the largest possible extent of the potential Vulcanoid region, and it shows the locations of the resonance instability zones derived by Evans and Tabachnik [19, 20]. The important mean-motion resonances that come into play are the 4:1, 3:1 and 2:1 resonances with Mercury and the 8:1 resonance with Venus.

\section{The Vulcanoid Zone Crossers}

This study is partially motivated by the possibility that as a cometary nucleus passes through the hypothesized Vulcanoid zone it might suffer an impact. The consequences of such an impact leading to observable imprints upon subsequent cometary behavior (e.g., through outbursts and fragmentation events) and/or, if applicable, distinct and unusual activity within associated meteor showers (e.g., through meteor storms or unusually high fireball activity). The most difficult problem with such an investigation is to differentiate between collisional fragmentation and nuclear fragmentation due to other mechanisms. This may be, for example, thermal or tidal fragmentation at the time of perihelion passage - these being processes that produce the same overall effect as a collision. Indeed, Belton [24] finds that the disruption rate for a $2 \mathrm{~km}$ diameter, active nucleus is of order $5 \times 10^{-5}$ per year irrespective of whether it passes through the Vulcanoid region or not. Indeed, we acknowledge that while the probability of observing the collisional disruption of a cometary nucleus during its passage through the Vulcanoid region is extremely small, it is not obviously, or necessarily zero.

Figure 2 shows the distribution of nodal points for those comets known to have passed within 0.06 to 0.25 au of the Sun. The orbital data used to construct figure 2 is taken from the JPL data archive for all known comets up to and including P/2014 E1 (Larson) - a catalogue that constitutes a total of 3261 sets of orbital elements. In all 406 recorded comets are found to have passed through the maximal Vulcanoid zone, the earliest recorded comet to do so being $\mathrm{C} / 400 \mathrm{~F} 1$, with comet $\mathrm{C} / 2008 \mathrm{~J} 13$ (SOHO) being the last in the data set being used. Among those objects qualifying for inclusion in figure 2 are numerous sungrazing comets, with the Kreutz, Kracht, Marsden and Meyer groups all being represented [25], a number of long period comets and two short period comets. Several of the comets in our figure 2 sample have shown past behaviors that seem worthy of further investigation, and table 1 provides a brief summary of their known properties. 


\section{Ecliptic crossing points}

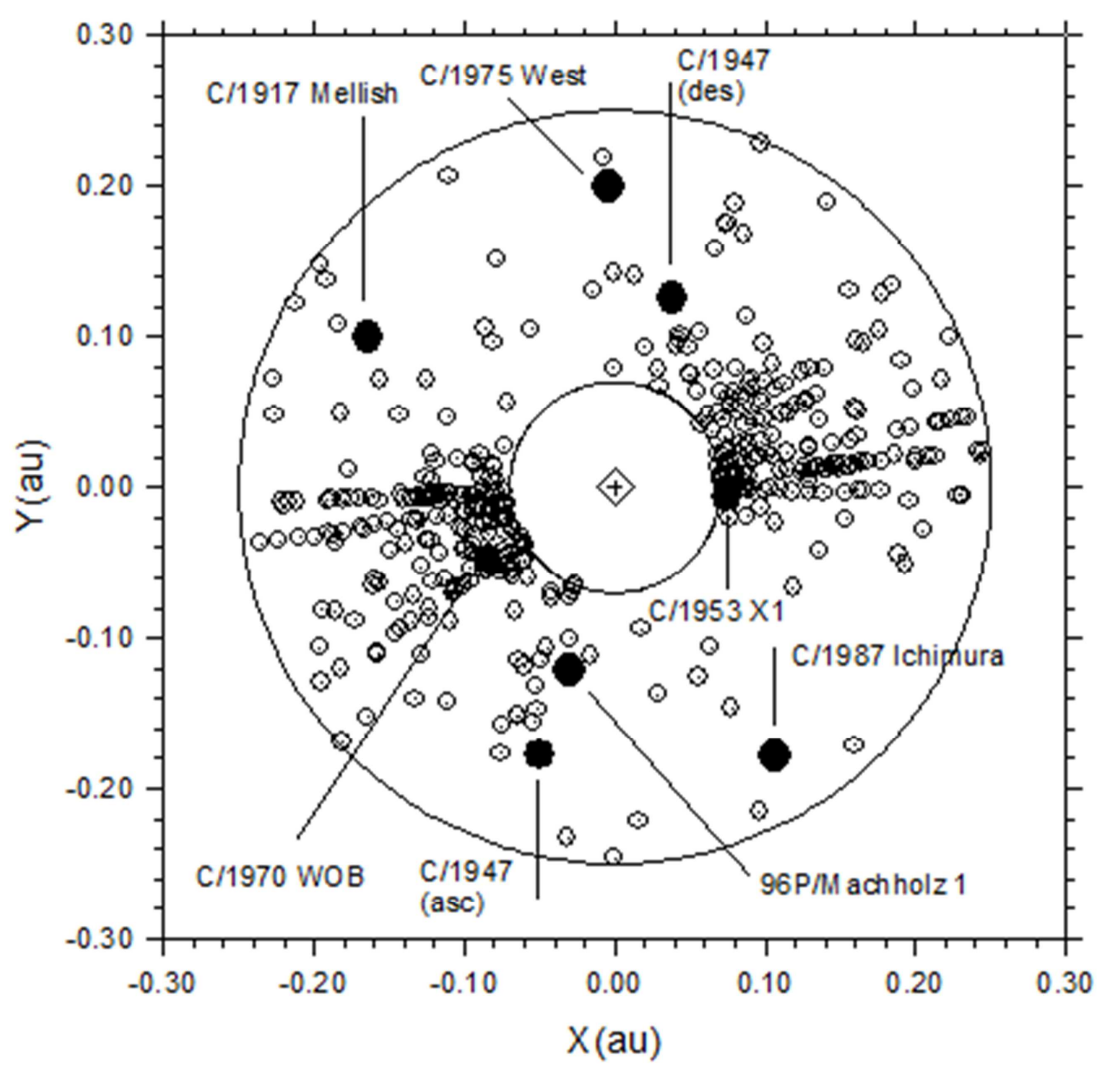

Figure 2. Distribution of cometary nodal-crossing points that fall within the maximum Vulcanoid region. The ascending and descending nodes for Kreutz sungrazing comets are clearly seen as two broad, fan-like features in the diagram. The comets highlighted by filled circles are described in table 1.

Table 1. Comets known to have passed at least once through the Vulcanoid region and that have either fragmented or have known associated meteor showers. Column 1 indicates the comet designation, column 2 is the perihelion distance and column 3 is a brief outline of the distinguishing characteristics.

\begin{tabular}{|c|c|c|}
\hline Comet & $q(\mathrm{au})$ & Behavior/associations \\
\hline $\mathrm{C} / 1769 \mathrm{P} 1$ & 0.124 & $\begin{array}{l}\text { Naked-eye comet; tail extended as much as } 70^{\circ} \text { on the sky [26] } \\
\text { Potential to produce a meteor shower on Mars [27] }\end{array}$ \\
\hline $\mathrm{C} / 1917 \mathrm{~F} 1$ & 0.190 & $\begin{array}{l}\text { Associated meteoroid streams }-11^{\text {th }} \text { century MON fireballs [28] } \\
\text { Potential to produce a meteor shower on Venus }[27,29]\end{array}$ \\
\hline C/ $1931 \mathrm{P} 1$ & 0.045 & Potential to produce meteor showers on Mars $[30,2]$ \\
\hline C/ 1947 X1 & 0.110 & $\begin{array}{l}\text { The "Great Southern Comet" } \\
\text { Nucleus fragmented post perihelion passage }\end{array}$ \\
\hline C/ $1953 \mathrm{X} 1$ & 0.072 & Nucleus fragmented \\
\hline C/ $1970 \mathrm{~K} 1$ & 0.009 & Nucleus split from a Kreutz sungrazing comet \\
\hline C/ 1975 V1 & 0.197 & Nucleus fragmented post perihelion passage \\
\hline C/ 1975 V2 & 0.219 & Association with the $\beta$ Tucanid meteoroid stream [2] \\
\hline C/ 1987 W1 & 0.200 & Nucleus fragmented \\
\hline C/ $1991 \mathrm{X} 2$ & 0.199 & $\begin{array}{l}\text { Lost post perihelion passage - nucleus disruption (?) } \\
\text { Outburst activity in } 1986\end{array}$ \\
\hline 96P / Machholz 1 & 0.124 & $\begin{array}{l}2 \text { small fragments observed ahead of nucleus in } 2012[31] \\
\text { Associated with Arietid and S. } \delta \text { Aquarid meteoroid streams [2] }\end{array}$ \\
\hline
\end{tabular}

Of the 11 comets listed in table 1,5 were observed to fragment close to perihelion, while 1 [C/1970 K1 WhiteOrtiz-Bolelli] split at an estimated heliocentric distance of order $100 \mathrm{au}$, which is suggestive of the nucleus having suffered a fracturing event during perihelion passage. Three comets [96P/Machholz 1, C/1917 F1 Mellish, and C/1975 V2 Bradfield] have associated meteor showers on Earth, and 3
[C/1769 P1 Messier, C/1917 F1 Mellish, and C/1931 P1 Ryves] potentially produce meteor showers on Venus and/or Mars [2, 27, 29, 30]. While the latter three candidates provide no historical observations, the growing fleet of in-orbit and on-ground observation platforms (in the case of Mars) portends that data on the long-term behavior of meteor showers, beyond those observed at the Earth's orbit, will 
eventually become available [2, 32]. Indeed, this situation was partially realized during the close passage of comet C/2013 A1 (Siding Spring) to Mars on 19 October 2014, when a whole suite of satellites (some in orbit about Earth, others in orbit about Mars) and two surface rovers were used to monitor the encounter.

Additionally, of the comets listed in table 1, only 5 have non-parabolic orbits, and while the second recorded return of comet Mellish is set for 2061, comet 96P/Machholz 1 has a short orbital period of 5.24 years, next rounding perihelion in 2017. While unusual fireball activity has been observed with respect to the December Mononcerotids, associated with comet Mellish (see below for details), no similar such activity has been documented with respect to either the Arietid or the Southern delta-Aquarid meteor showers associated with 96P/Machholz 1. The Arietids, however, are a daytime meteor shower that can only be studied through radio reflection or backscatter radar techniques. Consequently, while it is now known that the Arietids are one of the most intense of the annual meteor showers [33], very little is known about the shower's historical activity. Two small fragments were observed by the $\mathrm{SOHO}$ spacecraft to precede, by several hours, comet 96P/ Machholz 1 to and around perihelion in 2012, and it is presumed that these pieces broke away from the nucleus at the time of its perihelion passage in 2007. Such fragmentation events may occur on a regular basis as $96 \mathrm{P} / \mathrm{Machholz} 1$ rounds the Sun, but it is not currently clear how such events might moderate the expected activity from its associated annual meteor showers.

The vast majority of comets appearing in figure 2 are Kreutz sungrazing comets, and most were discovered with the coronagraph aboard the $S O H O$ spacecraft. The Kreutz comets almost always follow parabolic orbits, although there do appear to be sub-families of sungrazing comets having near identical orbital parameters, and there may also be periodic sungrazers. Indeed, of the 11 potential periodic sungrazers listed by Fernandez [34], 5 cut through the Vulcanoid zone: these are C/1999N5, C/1999 X3, C/2001 D1, C/2008 Q8 and C/2002 S11. All of these latter comets have estimated orbital periods between 4 and 6 years; periods very similar to that deduced for $96 \mathrm{P} / \mathrm{Machholz} 1$. Indeed, it has been suggested that comet $96 \mathrm{P} /$ Machholtz 1 is a member of the Marsden subgroup of sungrazers [35, 36]. Furthermore, we note that Sekhar and Asher [37] have shown that of the various sungrazing cometary groups, only the Marsden family can produce Earth-intercepting meteoroid streams at the present epoch. It has additionally been observed, however, that comet 96P/Machholz 1 has a particularly odd composition, being depleted in carbon-chain molecular species and cyanogens [38]. The distinct composition of $96 \mathrm{P} / \mathrm{Machholz} 1$ brings in to question its membership within the Marsden group, indicating, perhaps, that more work is required with respect to resolving the details of its specific origin, family association, and heritage. The Kreutz group of comets as well as the Marsden, Meyer and Kracht sungrazing subgroups [25] are most probably the result of an on-going cascade of fragmentation events precipitated by the break-up of a large parent nucleus some several thousands of years ago [36]. We note here that the possibility that the initial break-up event was triggered via a collision with a Vulcanoid asteroid cannot be ruled-out, or simply dismissed as being improbable, at the present time.

There is no specific evidence to suggest that any of the comets listed in table 1 fragmented as a result of a collision with a Vulcanoid or small (Vulcanoid derived) meteoroid fragment (although in principle it is a viable mechanism in each case), and only comet Mellish moves on an orbit that appears stable enough to have passed through the Vulcanoid zone numerous times in the past (see below for details). While comet 96P/Machholz 1 will have additionally made many passages through the Vulcanoid zone, a numerical integration study by Neslusan, Hajdukova and Jakubik [39] reveals that it has undergone a much more dramatic and more rapid orbital evolution than that exhibited by comet Mellish. Indeed, Ohtsuka, Nakano and Yoshikawa [35] note that 96P/Machholz 1 is in a 9:4 mean motion resonance with Jupiter, and that while the semi-major axis, and the direction of its apsides remains largely constant over time, its perihelion distance and orbital inclination can vary dramatically on a cyclical timescale of $\sim 3500$ years. More recently, high precision numerical integrations carried out by de la Fuente Marcos, de la Fuente Marcos and Aarseth [40] reveal that comet $96 \mathrm{P} / \mathrm{Machholz} 1$ is presently trapped within a Kozai resonance with Jupiter, with the inclination and eccentricity varying in opposite phase between $\sim 10$ and 80 degrees and $\sim 0.6$ and $\sim 1$, respectively. Additionally, however, the study by de la Fuente Marcos et al. [40] reveals that the orbit of comet $96 \mathrm{P} / \mathrm{Machholz} 1$ is chaotic on timescales longer that a few thousands of years. At the present time the impact probability for comet 96P/Machholtz 1 is an exceptionally small $5 \times 10^{-19}$ per perihelion passage (based upon the equations described in Section 4.2 below).

Of the 406 comets identified as having passed through the Vulcanoid zone at least once, the vast majority, 346 in fact, were discovered through the coronagraph instrument on the $\mathrm{SOHO}$ spacecraft [41]. The first comet discovered being $\mathrm{C} / 1996 \mathrm{D} 1$ and the last being C/2008 J13. The typical time that each of the $\mathrm{SOHO}$ comets spent in crossing the Vulcanoid zone is found, by straightforward orbital analysis, to be of order 1 day, with the typical path length of order 0.1 au. If it is assumed that the typical nucleus size of these comets is $1 \mathrm{~km}$, then the total volume swept out within the maximum Vulcanoid zone (figure 1) amounts to some $10^{-15}$ astronomical units cubed. This sampled space constitutes an extremely small fraction of the total 0.0167 astronomical units cubed volume of the maximum Vulcanoid zone and underscores the low probability of an actual collision and/or fragmentation event being observed at the present epoch. This result, however, is not the same as saying that collisions cannot occur, or have never occurred - it simply reflects the circumstances associated with the available, but known to be historically incomplete, dataset accumulated over a relatively short 12 year time interval. As time progresses and more and 
more sungrazer comets are discovered and followed, so the possibility of a collision with a Vulcanoid, however small that probability actually is, increases - presupposing, of course, that there is a Vulcanoid population with which cometary nuclei can interact with.

\section{Comet C/1917 F1 Mellish}

Discovered by John Mellish on 20 March 1917, comet C/1917 F1 Mellish, hereafter simply comet Mellish, is a relatively low-inclination, $i=32.68^{\circ}$, Halley family object with one of the smallest perihelion distances, $q=0.1902 \mathrm{au}$, of any known comet. Its orbital eccentricity is relatively high at $e=0.9931$, indicating an aphelion distance of $Q=55.10$ $\mathrm{au}$, and the deduced orbital period is 145.37 years. We note here that the orbit of comet Mellish is not known to the highest order of confidence, but for the analysis that follows we shall adopt the nominal JPL dataset values - indeed, these are the values that have just been presented. Based upon the recorded magnitude estimates, making $H_{0}=+7.4$, the comet's nucleus is given a provisional diameter of $3.1 \mathrm{~km}$ by Jenniskens [2]. Again, this value for the nuclear diameter is not well constrained, but we take it as a characteristic value in the analysis that follows in section 4.2. The nominal orbit of comet Mellish close to its perihelion point, as projected onto the ecliptic plane, is shown in figure 3.

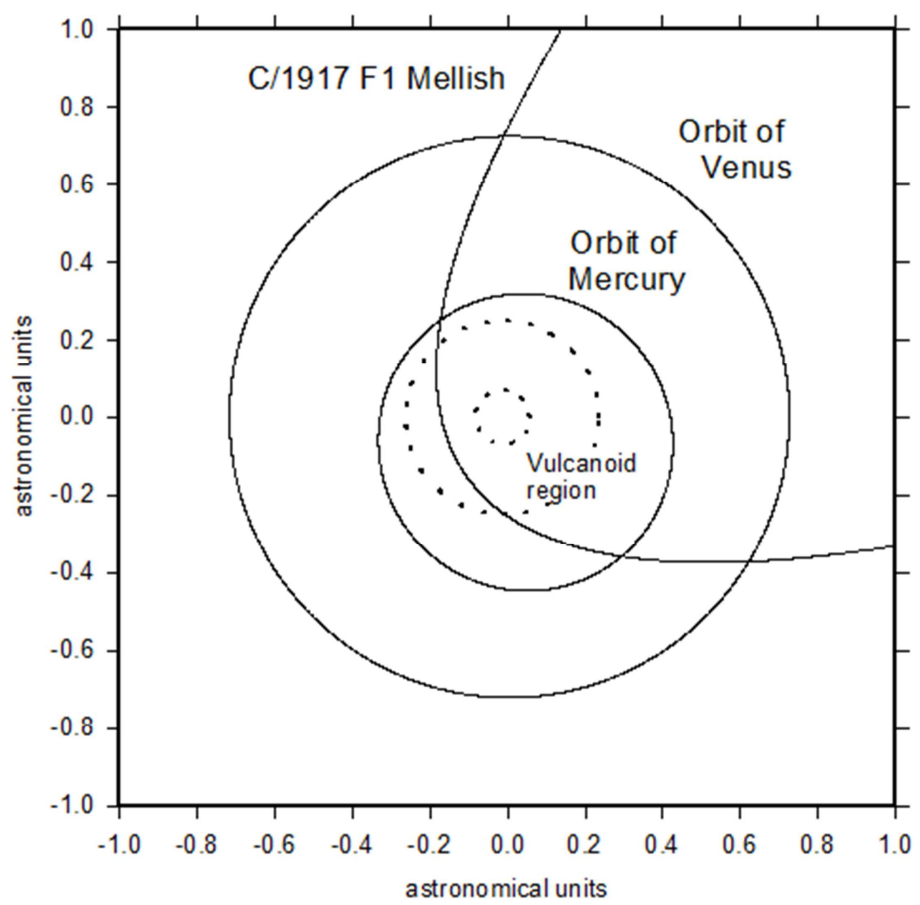

Figure 3. The inner solar system within 1 au of the Sun. The orbit of comet Mellish close to perihelion is shown, along with the orbits of Mercury and Venus. The two inner (dashed line) circles, with radii of 0.06 and 0.25 au respectively, indicate the approximate stability boundaries allocated to the maximum Vulcanoid region (see section 2 for details).

The ascending and descending nodal points of comet Mellish, at the present epoch, are located at 0.78 and $0.20 \mathrm{au}$ from the Sun, and accordingly when passing through perihelion the nucleus of comet Mellish skims the outer disk region of the Vulcanoid zone. In spite of having an ascending node that currently passes close to the orbit of Venus, the perihelion distance of comet Mellish has hardly changed during the past three millennia [42]. A longer-term numerical integration study conducted by Neslusan and Hajdukova [43] further indicates that the perihelion distance of comet Mellish has remained between 0.15 and 0.20 au for the past 40 millennia. Going back further in time, however, the perihelion distance increases quite rapidly, being of order 0.4 au some 50,000 years ago. In the same time interval the orbital inclination of comet Mellish has varied quite significantly, being some $25^{\circ}$ and close to its present-day value starting about 20,000 years ago; 50,000 years ago it was nearer to $55^{\circ}$. Comet Mellish has only been observed during one perihelion passage, and it has displayed no observed outburst activity. The comet, however, is known to be associated with several weak annual meteor showers (at the present epoch) and it is these that we describe in the next section.

\subsection{Comet Mellish Meteor Showers}

A number of annual meteor showers have orbits that indicate parentage to comet Mellish. The most robust association is that found by Whipple [44] who used the Harvard Super Schmidt Survey catalogue to establish a connection with the December Monocerotids (MON). This comet-stream parentage has been further strengthened through studies conducted by Lindblad and Olsson-Steel [45] and more recently by Veres, Kornos and Toth [42]. Although an annual meteor shower, the December Monocerotids deliver no more than a few visual meteors per hour at the time of shower maximum (located at a solar longitude of $\lambda=$ 
$\left.261^{\circ}\right)$. Veres, Kornos and Toth [42] have further suggested that comet Mellish is the parent of the November Orionids (NOO) which peak at a solar longitude of $\lambda=246^{\circ}$ with, again, a low zenith hourly rate of just 2 at shower maximum. Most recently, Neslusan and Hajdukova [43] have suggested that the April $\rho$ Cygnids (ARC), which peak at a solar longitude of $\lambda=37^{\circ}$, are additionally associated with comet Mellish. While Neslusan and Hajdukova found no clear evidence for a linkage between comet Mellish and the NOO in their study, they do suggest that the stream may have originated from a fragment that broke away from comet
Mellish, "a relatively long time ago". All three of these showers produce weak visual displays, but they are reasonably conspicuous in radar surveys, indicating a predominance of small, low mass meteoroids within each stream [46]. Indeed, the radar surveys indicate that the showers are relatively long-lived, with durations extend to 10, 32 and 10 days for the MON, NOO and ARC respectively. Figure 4 shows the orbits of comet Mellish along with the stream averaged orbits for the December Monocerotids, the November Orionids and the April $\rho$ Cygnids

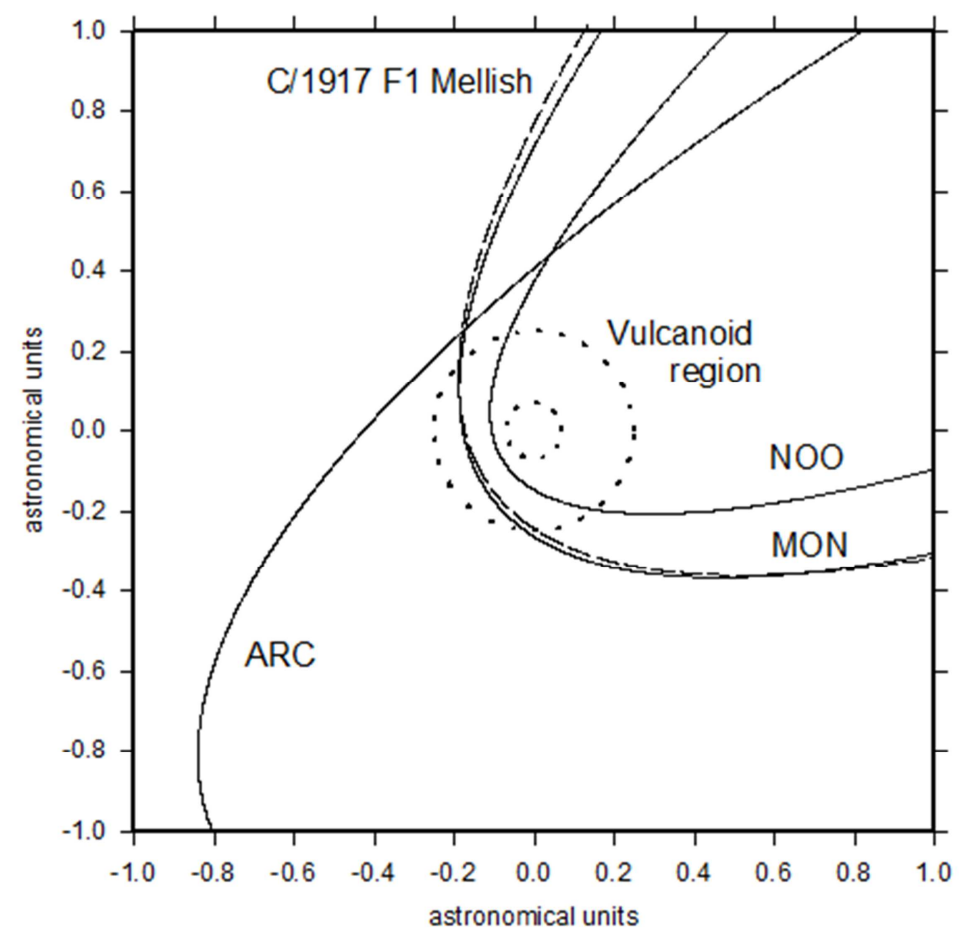

Figure 4. Stream averaged orbits for comet Mellish (dashed line) and its three associated meteoroid streams, the November Orionids (NOO), the December Monocerotids (MON) and the April $\rho$ Cygnids (ARC). Stream orbit data from [46]

While the similarity between the orbit of comet Mellish and those for the MON and NOO streams is indicative of these two streams being comprised of material ejected in the past 40,000 years, the linkage with the ARC stream requires ejection some 50,000 years ago, when the comet had a much higher orbital inclination and a much larger perihelion distance [43].

Historically, only the December Monocerotids appear to have produced any outburst activity, when the visual meteor rate and/or meteor characteristic were significantly different to the present day norm. Specifically, using the historical records of bright fireballs, Astapovich and Terenteva [28] have argued that a series of exceptionally bright fireballs were observed across Europe, in early December, over the interval from AD 1038 to 1099 . Furthermore, these fireballs were derived from a region that is consistent with an origin from MON radiant. Hasegawa [47] extended the analysis of Astapovich and Terenteva to include historic Chinese records and found that bright fireball activity could be linked to the MON radiant from as far back as AD 381 to as recently as
AD 1508. Fox and Williams [48] studied the possible origin of these ancient December fireballs and concluded that an association with the MON's was likely - they also noted that the orbits of the stream meteoroids, just like that of the parent comet, are evolving very slowly. Not only will the MON stream likely produce a shower at the Earth's orbit for at least the next millennium, it has likely produced a weak meteor shower on Earth for at least the past several thousands of years. This latter observation is suggestive of the possibility, therefore, that the extraordinary fireballs observed from the MON radiant in medieval times had an abnormal origin; that is, they might have been produced by an impact event upon of the parent comet. This idea builds upon the observation that fireball activity is not a normal characteristic of the present day MON shower, and that the epoch over which the fireballs were observed, lasting perhaps several hundreds of years, is short compared to the evolutionary timescale of the stream itself. If the meteoroids responsible for the fireballs seen in medieval times were produced as a result of a collision with the nucleus of comet Mellish, then the question 
arises as to the most likely solar system location at which the impact took place. The relatively high orbital inclination of the MON stream during the past 50,000 years [43], suggests that a collision with a main asteroid belt object is unlikely, and accordingly we might look for an alternative location where impacts could have occurred. In this manner, the small perihelion distance of Comet Mellish, as well as the MON stream, $q=0.1902$ and 0.1936 au respectively (at the present epoch), invites an investigation into the possibility of collisions with material located within the Vulcanoid zone.

\subsection{Impact probability and effects}

The probability of collision $P$ between a given comet and an object (e.g., asteroid, Vulcanoid, meteoroid) on a circular orbit of radius $R$ about the Sun can be evaluated via the equation derived by Kessler [49]. Accordingly,

$$
P=\frac{V_{r e l} \sigma t}{2 \pi^{3} R a \sqrt{\sin ^{2} i(R-q)(Q-R)}}
$$

Where $V_{\text {rel }}$ is the relative velocity at the time of encounter, $\sigma$ is the collisional cross-section area, $t$ is the time of potential encounter, $a$ is the semi-major axis of the comet's orbit, $i$ is the angle between the cometary orbit and the orbit of the impactor, with $q=a(1-e)$ and $Q=a(1+e)$ being the perihelion and aphelion distances of the comet, and $e$ is the comet's orbital eccentricity. We have assumed in equation (1) that the angle $\beta$ in Kessler's original equation, which accounts for the latitude variation in the impactor number density, is zero. The collision cross-section is taken to be the geometric cross-section with $\sigma=\pi\left(r_{\mathrm{c}}+r_{\mathrm{a}}\right)^{2}$, where $r_{\mathrm{c}}$ and $r_{\mathrm{a}}$ are the radii of the cometary nucleus and the impactor respectively. The relative velocity at the time of encounter is expressed via Opik's classic formula [50].

$$
V_{r e l}^{2}=\left(3-\frac{R}{a}-2 \sqrt{\frac{a}{R}\left(1-e^{2}\right)} \cos i\right) V_{c i r c}^{2}
$$

where $V_{\text {circ }}$ is the circular velocity at radius $R$. In the analysis that follows we look at the conditions that apply at the time just past perihelion passage and the arrival of comet Mellish at its inner most nodal point. Accordingly, the orbital radius of our putative impacting Vulcanoid is taken to be $R=$ $0.2 \mathrm{au}$, which dictates that $V_{\text {circ }}=59.6 \mathrm{~km} / \mathrm{s}$. Opik's formula for the relative velocity additionally gives, $V_{\text {rel }}=57.0 \mathrm{~km} / \mathrm{s}$. With the radius of comet Mellish being taken as $r_{\mathrm{c}}=1500$ meters, the collision probability evaluates to

$$
P=\left(3 \times 10^{-25}\right)\left(1+r_{a} / r_{c}\right)^{2} t
$$

The potential encounter time $t$ will be of order the time for comet Mellish to move through the Vulcanoid zone, and from straightforward orbital integration we find $t \approx 2.2$ days. With this characteristic time of potential encounter, the impact probability per perihelion passage of comet Mellish with a Vulcanoid of radius $r_{\mathrm{a}}$ is $P \approx 6 \times 10^{-20}\left(1+r_{\mathrm{a}} / r_{\mathrm{c}}\right)^{2}$.

Continuing in an order of magnitude vein, given the orbit of comet Mellish has not significantly changed over the past 40,000 years, as indicated by the calculations reported by Neslusan and Hajdukova [43], then the probability of an impact having occurred in the past 400 centuries is $P \approx 2 \times 10^{-}$ ${ }^{17}\left(1+r_{\mathrm{a}} / r_{\mathrm{c}}\right)^{2}$. This calculation indicates the impact probability, with a single Vulcanoid object, over what amounts to some 275 perihelion returns, is certainly extremely small. Reversing the argument, however, suggests that if an impact upon comet Mellish has occurred, within the allotted timeframe of 40,000 years, then the required number of potential impacting Vulcanoids in the region that comet Mellish physically samples must be of order $5 \times 10^{16}$ objects and this, of course, implies that they must be small fragments.

Steffl et al. [11] estimate from their analysis of STEREO spacecraft derived data that there are "no Vulcanoids larger than $5.7 \mathrm{~km}$ in diameter", and that, "there are no more than 76 Vulcanoids larger than $1 \mathrm{~km}$ " at the present epoch. For a collisionally evolved population of objects it is to be expected that there will be many smaller mass (sized) objects than larger ones. The number of objects larger than size $r$ within a given population of objects can generally be represented by a power law of the form $N(r)=N\left(r_{0}\right)\left[r / r_{0}\right]^{\alpha}$, where $r_{0}$ is a specific reference size and $\alpha$ is a constant of order -3.5 (appropriate to, say, a steady-state collisional fragmentation distribution [51]). The caveat with respect to the smaller Vulcanoid population, however, is that being situated so close to the Sun the thermally driven Yarkovsky effect will operate very efficiently and this will result in their rapid orbital evolution and ejection from the Vulcanoid zone [23]. Indeed, the high efficiency of the Yarkovsky effect will likely remove Vulcanoids smaller than $\sim 0.5 \mathrm{~km}$ across on a timescale shorter than the age of the solar system. Using the study of Steffl et al. [11] as our guide, however, it would appear than there might be no more than $\sim 900$ Vulcanoids with diameters between 0.5 and $2 \mathrm{~km}$ across. To accommodate the number of Vulcanoids needed to hypothetically generate a single collision with comet Mellish, the size distribution would need to be taken down to fragments as small as $5 \mathrm{~cm}$ in diameter. The existence of Vulcanoid fragments in this small size range can be fully ruled out given the apparent high efficiency of removal mechanisms. When combined with a model allowing for collisional evolution [22], however, in which a primordial population of large Vulcanoids is gradually broken down into smaller and smaller fragments, it might just be possible to maintain a relatively large number of small Vulcanoid fragments, but probably not in the required numbers to account for a single comet Mellish impact within the past 40,000 years. We note, however, that Stern and Durda [22] have argued that the most likely zone in which small Vulcanoids might best survive the ravages of impacts, as well as the effects of Yarkovsky clearing, is that region close its outer most boundary at 0.2 au and this is exactly the region that comet Mellish has regularly passed through. If any presently known periodic comet is going to show signs of interacting with small Vulcanoid fragments then it is most likely comet Mellish. In addition, and in a similar vein, Campins et al. [21] place their 'most survivable zone' for the 
Vulcanoids in the range between $\sim 0.08$ to 0.15 au from the Sun, which is the same region presently 'sampled' by comet 96P/Machholtz 1.

The consequences of an impact upon the nucleus of comet Mellish are difficult to fully gage. It is clear that the impact speed will be very high, and, as seen above, of order $57 \mathrm{~km} / \mathrm{s}$. Results from the Deep Impact mission [52] indicate that about $10^{6} \mathrm{~kg}$ of nuclear material was excavated and released from comet 9P/Tempel 1 following the impact of a $372 \mathrm{~kg}$ copper cylinder at a velocity of $10.3 \mathrm{~km} / \mathrm{s}$. The same kinetic energy of impact would be delivered by a $12 \mathrm{~kg}$ object at 57 $\mathrm{km} / \mathrm{s}$ - this corresponds to an object of just 20 centimeters across (assuming a density of $3300 \mathrm{~kg} / \mathrm{m}^{3}$ ). However, our size distribution of interest with respect to comet Mellish includes objects as small as $5 \mathrm{~cm}$ across. At this size the impact energy of a Vulcanoid fragment would be about $3.5 \times 10^{8}$ Joules - which is some 57 times smaller than that of the Deep Impact cylinder. This latter result continues to work against the possibility of a Vulcanoid collision induced origin for the December Monocerotid fireballs. Not only is the existence of a substantial $5 \mathrm{~cm}$ sized fragment population within the Vulcanoid zone highly unlikely, at the present epoch, but the observable consequences of any such collision would, it appears, be hardly noticeable.

Assuming a uniform solid water-ice nucleus, the gravitational binding energy of comet Mellish is of order $10^{13}$ Joules, and accordingly a collision with any object larger than about 15 meters across would likely be fully disruptive of the nucleus. As indicated in section 2 above the expected size range for present-day Vulcanoids falls between 0.5 and 6 $\mathrm{km}$, and as such under direct, head-on collision conditions the nucleus of comet Mellish would be completely destroyed. Given the actual existence of comet Mellish at the present time, however, one might argue that, at worst, a grazing or off-center collision with a Vulcanoid might have taken place in the past. This latter suggestion, however, is not convincing, and given that the ancient December Monocerotid fireballs really were the result of an abrupt influx of larger meteoroids into the stream, then the most likely explanation for that influx is nuclear fragmentation caused by thermal and/or tidal stress at the time of perihelion passage.

\section{Conclusions}

The observational evidence (as illustrated in figure 2) reveals that there is a great deal of dynamical activity within the hypothesized Vulcanoid zone (recall figure 1) - comets, as the $\mathrm{SOHO}$ coronagraph data indicates, continuously passing through the region on an almost daily basis [53]. The Vulcanoid zone is not only dynamically active it is also a region where any passing cometary nuclei will be placed under considerable tidal and thermal stress. If any of the cometary nuclei identified within this study are going to fragment then the most-likely place that they will do so is at perihelion [24, 54], and this will make the identification of any Vulcanoid (or small Vulcanoid fragment) impact more difficult to resolve. It is not our intention to suggest that all or indeed any of the close-to-perihelion fragmentation events (as described in table 1) that have occurred for those comets known to have passed through the Vulcanoid zone are the result of collisions, but the possibility cannot, out of hand, be entirely written-off. Certainly, the odds are very much against any such collisions taking place at the present epoch, but they are not automatically zero. Of all the comets identified as having entered the Vulcanoid zone more than once, comet C/1917 F1 Mellish has proved to be the most interesting. There is no compelling evidence, however, to indicate that the anomalous fireball activity identified with respect to its associated meteoroid stream (the December Monocerotids) in the $11^{\text {th }}$ Century was due to a collision suffered within the putative Vulcanoid zone.

As with earlier investigations, by other researchers, into the possible presence of Vulcanoid asteroids within the inner solar system, at the present epoch, we find no compelling evidence to betray their existence on the basis of historically recorded cometary behaviors. Indeed, it is not known if any Vulcanoid asteroids have ever existed within our solar system, but this fact alone provides every reason and motivation to continue the search for such objects, and any evidence that implies their past existence.

\section{References}

[1] Taylor, M. G. G. et al. 2015. Rosetta begins its comet tale. Science, 347, 387.

[2] Jenniskens, P. 2006. Meteor Showers and their Parent Comets. CUP, Cambridge.

[3] Hsieh, H. H, and Jewitt, D. 2006. A population of comets in the main asteroid belt. Science, 312, 561 .

[4] Bertini, I. 2011. Main belt comets: a new class of small bodies in the solar system. Plan. Space Sci. 59, 365.

[5] Hainaut, O. R., et al. 2012. P/2010 A2. I: an impact in the asteroid belt. A\&A. 537, A69.

[6] Beech, M., and Gauer, K. 2002. Cosmic roulette: comets in the main belt asteroid region. EMP. 88, 211.

[7] Campbell, W. W., and Trumpler, R. 1923. Search for intramercurial bodies. PASP. 35, 214.

[8] Baum, R., and Sheehan, W. 1997. In Search of Planet Vulcan, the ghost in Newton's clockwork machine. Plenum Press, New York.

[9] Durda, D. D., et al. 2000. A new observational search for Vulcanoids in SOHO/LASCO coronagraph images. Icarus, 148,312 .

[10] Schumacher, G., and Gay, J. 2001. An attempt to detect Vulcanoids with $S O H O / L A S C O$ images I. Scale relativity and quantization of the solar system. A\&A. 368, 1108.

[11] Steffl, A. J., Cunningham, N. J., Shinn, A. B., Durda, D. D., and Stern, S. A. 2013. A search for Vulcanoids with the STEREO heliosphere imager. Icarus, 223, 48.

[12] Hughes, D. W. 1990. Cometary outbursts: a review. Q. J. R. Astr. Soc. 31, 69. 
[13] Gronkowski, P. 2002. Outbursts of comets - the case of 1P/Halley. Plan. Space Sci. 50, 247.

[14] Boehnhardt, H. 2004. Split comets. In Comets II, M. C. Festou, H. U. Keller, and H. A. Weaver (Eds.), University of Arizona Press, Tucson. p. 301.

[15] Courten, H. C., Brown, D. W., and Albert, D. B. 1976. Summary paper: ten years of solar eclipse comet searches. BAAS. 8, 504.

[16] Swift, L. 1878. Discovery of Vulcan. Nature, 18, 539.

[17] Merline, W. J., et al. 2008. A program to search for Vulcanoids from MESSENGER. BAAS. 40, 491.

[18] Chapman, C. R. et al. 2008. First MESSENGER insights concerning the early cratering history of Mercury. www.lpi.usra.edu/meetings/bombardment2008/pdf/3014.pdf.

[19] Evans, N. W., and Tabachnik, S. A. 1999. Possible long-lived asteroid belts in the inner solar system. Nature, 399, 41

[20] Evans, N. W., and Tabachnik, S. A. 2002. Structure of possible long-lived asteroid belts. MNRAS. 333, L1.

[21] Campins, H., Davis, D. R., Weidenschilling, S. J., and Magee, M. 1996. Searching for Vulcanoids. In Completing the Inventory of the Solar System, T. W. Rettig and J. M. Hahn (Eds.), ASP Conference Series, 107, p. 85.

[22] Stern, S. A., and Durda, D. D. 2000. Collisional evolution of the Vulcanoid region: implications for present-day population constraints. Icarus, 143, 360.

[23] Vokrouhlicky, D., Farinella, P., and Bottke, W. F. 2000. The depletion of the putative Vulcanoid population via the Yarkovsky effect. Icarus, 148, 147.

[24] Belton, M. J. 2015. The mass distribution of Jupiter family comets. Icarus, 245, 87.

[25] Marsden, B. 2005. Sungrazing comets. ARAA. 43, 75.

[26] Kronk, G. W. 1999. Cometography - a catalogue of comets volume 1: ancient-1799. CUP, Cambridge.

[27] Christou, A. A. 2010. Annual meteor showers at Venus and Mars: lessons from the Earth. MNRAS. 402, 2759.

[28] Astapovich, I. S., and Terenteva, A. K. 1968. Fireball radiants appearing between the $1^{\text {st }}$ and $15^{\text {th }}$ centuries. In Physics and Dynamics of Meteors. L. Kresak and P. M. Millman (Eds.), Reidel, Dordrecht. p. 308.

[29] Beech, M. 1998. Venus-intercepting meteoroid streams. MNRAS. 294, 259.

[30] Treiman, A. H., and Treiman, J. S. 2000. Cometary dust streams at Mars: preliminary predictions from meteor streams at Earth from periodic comets. J. Geophys. Res. 105, 24,571.

[31] http://sungrazer.nrl.navy.mil/index.php?p=news/machholz_ba bies.

[32] Beech, M., and Brown, P. 1995. On the visibility of bright Venusian fireballs from Earth. EMP. 68, 171.

[33] Campbell-Brown, M. 2004. Radar observations of the Arietids. MNRAS. 352, 1421

[34] Fernandez, Y. R. 2009. That's the way the comet crumbles: splitting Jupiter-family comets. Plan. Space Sci. 57, 1218.
[35] Ohtsuka, K., Nakano, S., and Yoshikawa, M. 2003. On the association among periodic comet $96 \mathrm{P} / \mathrm{Machholz}$, Arietids, the Marsden comet group, and the Kracht comet group. Publ. Astron. Soc. Japan, 55, 321.

[36] Sekanina, Z., and Chodas, P. 2007. Fragmentation hierarchy of bright sungrazing comets and the birth and orbital evolution of the Kreutz system II. The case for cascading fragmentation. ApJ. 663, 657

[37] Sekhar, A., and Asher, D. J. 2014. Meteor showers on Earth from sungrazing comets. MNRAS. 437, L71.

[38] Schleicher, D. G. 2008. The extremely anomalous molecular abundances of comet 96P/Machholz 1 from narrowband photometry. Astron. J. 136, 2204.

[39] Neslusan, L., Hajdukova, M., and Jakubik, M. 2013. Meteorshower complex of asteroid $2003 \mathrm{EH} 1$ compared with that of comet 96P/Machholz. A\&A. 560, id A47.

[40] De la Fuente Marcos, C., de la Fuente Marcos, R, and Aarseth, S. J. 2015. Flipping minor bodies: what comet 96P/Machholz 1 can tell us about the orbital evolution of extreme transNeptunian objects and the production of near-Earth objects on retrograde orbits. MNRAS. 446. 1867.

[41] Biesecker, D. A., Lamy, P., St. Cyr, O. C., Llebaria, A., and Howard, R. A. 2002. Sungrazing comets discovered with the SOHO/LASCO coronagraphs 1996- 1998. Icarus, 157, 323.

[42] Veres, P., Kornos, L., and Toth, J. 2011. Meteor showers of comet C/1917 F1 Mellish. MNRAS. 412, 511.

[43] Neslusan, L., and Hajdukova, M. 2014. The meteor-shower complex of comet C/1917 F1 (Mellish). A\&A. 566, A33.

[44] Whipple, F. 1954. Photographic meteor orbits and their distribution in space. Astron. J. 59, 201.

[45] Lindblad, B. A., and Olsson-Steel. D. 1990. The Monocerotid meteor stream and comet Mellish. Bull. Astron. Inst. Czechosl. 41, 193.

[46] Brown, P., Wong, D., Weryk, R., and Wiegert, P. 2010. A meteoroid stream survey using the Canadian Meteor Orbit Radar II: identification of minor showers using a 3D wavelet transform. Icarus, 207, 66.

[47] Hasegawa, I. 1999. Historical meteor showers - Geminids and December Monocerotids. In Meteoroids 1998, W. J. Baggaley and V. Porubcan (Eds.), Astron. Inst. Slovak Acad. Sci. Bratislava. p.177.

[48] Fox, K., and Williams, I. P. 1985. A possible origin for some ancient December fireballs. MNRAS. 217, 407.

[49] Kessler, D. 1981. Derivation of the collision probability between orbiting objects: the lifetime of Jupiter's outer moons. Icarus, 48, 39.

[50] Opik, E. 1951. Collision probability with the planets and the distribution of planetary matter. Proc. Roy. Irish Acad. 54A, 165 .

[51] Dohnanyi, J. S. 1972. Interplanetary objects in review: statistics of their masses and dynamics. Icarus, 17, 1.

[52] A'Hearn, M. F., et al. 2005. Deep Impact: excavating comet Tempel 1. Science, 310, 258. 
[53] Sekanina, Z. 2002. Statistical investigation and modeling of sungrazing comets discovered with the Solar and Heliospheric Observatory. Ap. J. 566, 577.
[54] Chen, J., and Jewitt, D. 1994. On the rate at which comets split. Icarus, 108, 265. 